\title{
Erratum:
}

\section{Vol. 69, No. 12}

In the report "Tuberculosis Preventive Treatment Scale-Up Among Antiretroviral Therapy Patients - 16 Countries Supported by the U.S. President's Emergency Plan for AIDS Relief, 2017-2019," on page 329, an author's academic degree was listed incorrectly. The correct degree is Sevim Ahmedov, MD. 